\title{
Laboreal
}

Volume $16 \mathrm{~N}^{\circ} 2$ | 2020

Programa de Pesquisa do Curso da Ação

\section{A ergotoxicologia, da palavra aos conceitos e às metodologias}

La ergotoxicología, de la palabra a los conceptos y metodologías

L'ergotoxicologie, du mot aux concepts et méthodologies

Ergotoxicology, from the word to concepts and methodologies

\section{Louis Galey e Alain Garrigou}

\section{(2) OpenEdition}

Journals

Edição electrónica

URL: http://journals.openedition.org/laboreal/16406

DOI: 10.4000/laboreal.16406

ISSN: 1646-5237

\section{Editora}

Universidade do Porto

Refêrencia eletrónica

Louis Galey e Alain Garrigou, "A ergotoxicologia, da palavra aos conceitos e às metodologias », Laboreal [Online], Volume 16 №2 | 2020, posto online no dia 01 dezembro 2020, consultado o 14 dezembro 2020. URL : http://journals.openedition.org/laboreal/16406 ; DOI : https://doi.org/10.4000/ laboreal.16406

Este documento foi criado de forma automática no dia 14 dezembro 2020.

Laboreal está licenciado com uma Licença Creative Commons - Atribuição-NãoComercial 4.0 Internacional. 


\section{A ergotoxicologia, da palavra aos conceitos e às metodologias}

La ergotoxicología, de la palabra a los conceptos y metodologías

L'ergotoxicologie, du mot aux concepts et méthodologies

Ergotoxicology, from the word to concepts and methodologies

Louis Galey e Alain Garrigou

\section{NOTA DO EDITOR}

Tradução : João Viana Jorge - joaojorg@gmail.com

\section{Robert Villatte e a inscrição da ergotoxicologia no projeto de uma ergonomia da atividade}

1 Em França, quando o livro "Les risques du travail. Pour ne pas perdre sa vie à la gagner" (Os riscos do trabalho. Para não perder a vida a ganhá-la) aparece em 1985 (Cassou et al, 1985), tinham passado apenas três anos sobre as leis Auroux que instauravam o "Comité d'Hygiène, Sécurité et des Conditions de Travail”, com novas prerrogativas no domínio das condições de trabalho nas empresas. Estas evoluções regulamentares traduzem as preocupações sociais a respeito do trabalho e da saúde. Nesse período a obra coordenado por médicos, médicos do trabalho e inspetores do trabalho, foi introduzida pelo director geral do "Institut national de la santé et de la recherche médicale (Inserm)"“ e o posfácio foi redigido por Alain Wisner. A saída do livro foi um evento nacional englobando 133 artigos e 106 autores entre os quais ergónomos, médicos e médicos do trabalho, psiquiatras, especialistas da estatística, docentes investigadores e engenheiros nomeadamente de instituições tão diversificadas como o "Institut National de Recherche et de Sécurité (INRS)", as "Caisses Régionales d'Assurance Maladie (CRAM)", designadas hoje por "CARSAT", o "Institut National de Recherche 
Agronomique (INRA)", o “Conservatoire national des arts et métiers (Cnam)", o "Organisme Professionnel de Prévention du Bâtiment et des Travaux Publics (OPPBTP) ", o “ Inserm ", o “ Centre national de la recherche scientifique (CNRS)" e o ministério do trabalho.

2 Nesta obra, um texto de três páginas, intitulado "Toxicologie et ergonomie" de Robert Villatte (1985), manter-se-á fundador em todo um campo de pesquisas e intervenções orientadas para a temática do trabalho na presença de produtos químicos. Foi a primeira vez em que se utilizou o termo "ergotoxicologia ", um eco do texto precursor de Delvolvé (1984), publicado no ano anterior acentuando, a importância de considerar a particularidade das atividades de trabalho em medidas para modular os efeitos das exposições sobre a saúde.

3 Robert Villatte, nascido em 1946, após uma formação técnica em física, trabalha na indústria farmacêutica e torna-se sindicalista militante na Confédération française démocratique du travail (CFDT - Fédération de la chimie). No quadro dos seus mandatos, como responsável sindical, depressa se interessa pelas consequências da exposição dos trabalhadores (por turnos) aos produtos tóxicos. Levado a colaborar com investigadores daquele domínio segue uma formação de ergónomo em dedicação exclusiva no Conservatoire national des arts et métiers (Cnam) organizada pela equipa dirigida por Alain Wisner (1923-2004) e efectua as suas primeiras pesquisas no terreno. Como aponta Charles Gadbois (1937-2019), na apresentação da entrevista realizada pela comissão Histoire de la Société d'Ergonomie de Langue Française (SELF), “ o seu percurso é balizado pela organização de formações em ergonomia no quadro sindical e a capitalização de saberes através da publicação de livros e artigos de revista" (Teiger et al., 2003). o texto "Toxicologie et ergonomie" inscreve-se neste contexto. Nele Villatte desenvolve a abordagem programática da ergotoxicologia face a múltiplas constatações :

- " Considerar que existe um " Homem médio " com reacções médias entra em contradição com os conhecimentos adquiridos sobre a dispersão dos parâmetros fisiológicos numa população de indivíduos e a sua evolução no decurso da vida " (p. 301) ;

- O impacto dos produtos tóxicos na saúde varia em função de numerosos factores (características do ambiente, variação do estado e mistura de poluentes nas oficinas, natureza do trabalho, atividade desenvolvida pelo trabalhador, etc.) ;

- É necessário definir as prioridades de prevenção em função das atividades do trabalho.

Para Villatte o simples respeito das normas não permite garantir a ausência de atentados à saúde. A evolução dos conhecimentos sobre a periculosidade dos novos produtos presentes no local de trabalho, a sua diversidade, as multiexposições, os protocolos e os processos de elaboração de normas aos quais se acrescenta a impossibilidade de ter em conta a singularidade das atividades de trabalho, quer nos estudos epidemiológicos quer nos toxicológicos, vêm acentuar a atualidade desse texto.

Atualmente as estratégias de prevenção constituídas em torno do uso de normas reforçaram-se. Face aos riscos profissionais que têm tendência a aumentar (DARES, 2019 ; Eurofound, 2017) um dos pilares da prevenção da exposição, por inalação, aos produtos químicos apoia-se no controlo da atmosfera do (local de) trabalho pela 
medida, por comparação com os valores limite de exposição profissional. Esses valores de referência são elaborados a partir da peritagem científica independente da l'Agence nationale de sécurité sanitaire (Anses) procurando identificar os limiares de exposição a partir dos quais os efeitos indesejáveis se observariam. o ministério da tutela do trabalho estabelece uma sua proposta do valor limite num projecto regulamentar. Finalmente esses valores são submetidos por concertação social às instâncias do Conseil d'Orientation des Conditions de Travail (COCT) sob a égide do ministério do trabalho (INRS \& ISSA, 2016). Neste contexto as normas relativas à toxicidade dos produtos são sempre elaboradas por compromissos, a partir de conhecimentos científicos, de contextos industriais e socioeconómicos (Deveau et al., 2015). o exemplo histórico do amianto (Henry, 2000), tal como as diferenças em função dos países e revisões regulares dos valores limite (a reduzir), lembram o potencial desfasamento entre os conhecimentos científicos dos efeitos na saúde e a regulamentação.

\section{A ergotoxicologia atual}

6 A ergotoxicologia define-se como uma prática particular da ergonomia (Garrigou, 2011) visando analisar as atividades de trabalho em meios profissionais em que as exposições a produtos químicos podem ocorrer. Neste quadro, a ergotoxicologia desenvolve “ modelos operativos (no sentido que lhes dá Wisner, 1972), ferramentas e meios de prevenção eficazes para gerir e prevenir os riscos para a saúde dos trabalhadores expostos a produtos químicos " (Garrigou, 2011, p. 25). Nesta aceção trata-se mesmo duma forma de pesquisa e de intervenção de intenção operacional profundamente ancorada na ergonomia. Pelos seus objetivos a ergotoxicologia mobiliza em complemento competências e modelos oriundos da toxicologia, da medicina do trabalho e da prevenção. Atualmente a ergotoxicologia não é apenas reservada aos ergónomos. É também mobilizada pelos agentes de prevenção, ergónomos e toxicólogos internos ou de serviço à saúde no trabalho (Mohammed-Brahim et al., 2018) alimentando uma prática transprofissional.

7 Os fundamentos históricos são os da análise situada das atividades (Leplat \& Cuny, 1977 ; Daniellou, 2005) e das abordagens culturalistas da segurança nas organizações (Morel et al., 2008), tendo em conta as representações dos riscos individual e socialmente construídos na ação (Duclos, 1987 ; Weill-Fassina, Rabardel \& Dubois, 1993), numa orientação de conceção dos sistemas de trabalho (Jeffroy, 1987; Garrigou et al., 1995). A análise da atividade de tabalho como ponto de partida (Garrigou, 2011) para retomar as exposições é uma distinção essencial da higiene industrial (Zartarian et al., 2004). Deste modo a exposição torna-se um objeto fronteira (Vinck, 2009) para animar as discussões e permutas entre profissões e disciplinas do âmbito da saúde no trabalho.

Dando sequência ao texto de Villatte, Sznelwar (1992) aplica a ergotoxicologia em grande escala a propósito da exposição dos agricultores aos pesticidas. Esta tese, sob a direção de Alain Wisner, mostra o carácter operacional da proposta de Villatte. Os resultados afirmam que a organização do trabalho e as estratégias dos operadores são aspetos fundamentais da exposição, que produtores e operadores elaboram compromissos e que é possível diferenciar a exposição em função das atividades desenvolvidas em cada etapa de trabalho. Esse trabalho permite uma comparação entre a França e o Brasil na linhagem da antropotecnologia (Wisner, 1985), aprofundando a importância da dimensão psicológica dos trabalhadores a partir da psicodinâmica do trabalho. 
O retomar das pesquisas na agricultura (Mohammed-Brahim et al., 1997) e a implementação da abordagem ergotoxicológica nos trabalhos de retirada de amianto (Mohammed-Brahim et al., 1998) permitiu reforçar a demonstração dos limites de um modelo por barreira de proteção (Mohammed-Brahim \& Garrigou, 2009), quer este se considere normativo (por aplicação de valores limite ou de restrições ao uso) baseado nas proteções e instruções (em torno do uso dos EPI - equipamentos de proteção individual - e de procedimentos a respeitar) ou médico (respeitante às aptidões $\mathrm{e}$ indicações médicas). Estes trabalhos do âmbito da agricultura interessaram-se precisamente pela ausência de eficácia da combinação de proteções em situações reais de adubagem em viticultura (Garrigou et al., 2011). Nesta continuidade, a demonstração de que o processo de homologação dos pesticidas se apoia na prescrição do uso de EPI ineficiente em certas situações de trabalho reforça o repor globalmente em causa as estratégias de prevenção por barreira de proteção (Garrigou et al., 2020). O paralelo entre as "barreiras de proteção" descritas por Mohammed-Brahim \& Garrigou (2009), e as "normas" questionadas por Villatte (1985) é inevitável. Esta demonstração no campo dos pesticidas ratifica a força da proposição inicial de Villatte. Todo um paradigma de prevenção funcionalista promulgado pelos human factors (Reason, 1990, por exemplo) é então reposto em causa [ $\left.{ }^{1}\right]$.

Nessa continuidade a construção de uma lógica de prevenção pôde ser desenvolvida a partir das pesquisas sobre segurança industrial (ver nomeadamente Morel et al.,2008; Daniellou et al., 2011; Nascimento et al., 2014 ; Rocha et al., 2015), permitindo pôr à prova as prescrições de prevenção. $O$ desenrolar deste modelo fez-se principalmente nas indústrias químicas de risco (Garrigou et al., 2015 ; Galey et al., 2017 ; MohammedBrahim et al., 2018 ; Galey, 2019) articulando a análise das situações reais de exposição (segurança gerida) para as confrontar com as prescrições de prevenção (segurança regulamentada) com o objetivo de desenvolver coletivamente recursos de segurança construídos [2]. Ainda que este modelo apresente limitações quando se trata de distinguir o regulamentado do gerido, do construído por exemplo, a sua operacionalização em método de pesquisa intervenção mostrou o seu interesse para debater as regras de segurança face à especificidade das situações de trabalho, como reivindicado por Villatte, as exposições vivenciadas pelos trabalhadores e as representações dos riscos associados, em paralelo com o funcionamento sistémico das organizações.

11 O cerne da ergotoxicologia prende-se assim com a resolução dos enigmas (Teiger, 1993), conducentes à compreensão das exposições profissionais a partir da análise da atividade (Garrigou et al., 2004; Mohammed-Brahim \& Garrigou, 2009). Os atores da empresa vão dispor de fragmentos deste enigma que deverão ser agrupados para (se) compreender a exposição. Essa compreensão passa por uma moblilização e uma colaboração estreita, similar sob certos aspetos a um inquérito, com os múltiplos atores internos ou exteriores à empresa, sejam os operadores, os quadros diretos ou longínquos, os responsáveis pela produção ou pelo serviço de investigação e desenvolvimento, o médico do trabalho, etc. Trata-se de fazer coexistir e compreenderem-se diferentes "mundos" (Béguin, 2004, 2010) possuidores dos seus próprios sistemas de "coordenadas profissionais" dependentes da maneira de pensar, de viver e de apreender as situações de trabalho (Judon, 2017). A atividade e os seus traços devem ser postos a circular no seio dos meios profissionais a fim de compreender as suas causas a partir dos elementos subjetivos e objetivos trazidos pelos diferentes 
atores (Garrigou et al., 2004). Essas reflexões sobre a intervenção alimentaram um modelo de intervenção ergotoxicológico (Mohammed-Brahim, 2015).

Algumas medidas são frequentemente utilizadas para reforçar uma compreensão global da atividade. Podem ser fisiológicas (frequência cardíaca, respiratória, medidas angulares...) ou químicas (concentração dos poluentes em tempo real, recolha de amostras em pachos ou filtros para análises físico químicas). Uma observação surpreendente, associada ao uso das medições quando estas estão associadas à descrição da atividade, é o modo como esta ferramenta permite estimular os debates sobre as atividades de trabalho, as representações e as práticas de prevenção (Galey, 2019). A integração das medições (pela técnica de video exposure monitoring por exemplo) numa estratégia de intervenção torna-se um suporte de transformação (Galey, Judon, Jolly et al., 2019).

13 Uma particularidade da ergotoxicologia é a de se apoiar numa diversidade de objetos intermediários (Vinck, 2009) para fazer emergir as atividades e a representação de risco de exposição. Esses objetos intermediários tomam a forma de medições, vídeo da atividade, procedimentos de segurança, etc. $\mathrm{O}$ seu uso nos "espaços intermediários de prevenção " (Judon, 2017 ; Judon et al., 2019) permite assim uma outra compreensão das situações de exposição através de uma abordagem reflexiva em que as dimensões íntimas do trabalho e da exposição, contribuindo para estabelecer uma ligação entre a vida no e fora do trabalho, são partilhadas entre os atores em presença. Nessas condições torna-se possível revelar os conhecimentos detalhados dos trabalhadores a respeito das situações de exposição graças aos objetos intermediários. Os desenvolvimentos metodológicos das entrevistas de confrontação (Mollo \& Falzon, 2004), associando a medição em tempo real ao vídeo, são particularmente demostrativos quanto a este ponto (Judon et al., 2019). o lugar das representações dos riscos torna-se a chave para analisar as estratégias dos operadores e alimentar as demarches de prevenção (Judon, 2017; Judon et al., 2015). Os equipamentos de protecção individual, por exemplo, constituem-se em objeto de discussão no seio dos coletivos de trabalhadores permitindo descrever as contradições e compromissos implementados nas estratégias de proteção como formas de minimização (Duclos, 1987) e de negação do risco, ou ainda dos limites da prescrição de prevenção descendente (Goutille et al., 2016).

\section{Para concluir : para uma conduta de projeto de conceção da prevenção}

14 Esta orientação, em progresso, desde os anos 2015, para a conceção parte da constatação de uma focalização no diagnóstico das situações de exposição, sem se alimentar da ergonomia de conceção, para reforçar o potencial de transformação.

Os últimos trabalhos em ergotoxicologia interessam-se pois pelos novos poluentes cujos perigos para a saúde são caracterizados pela incerteza (por exemplo as nanopartículas Galey, Audignon, Brochard et al., 2020 - e o betume no quadro dos trabalhos rodoviários), mas pesquisando as possibilidades de desenvolver projetos de conceção da prevenção (Judon, 2017; Galey, 2019). Este contexto de incerteza induz a ausência de "normas" admitidas de exposição, específicas desses produtos químicos. Um objetivo 
central é o de reduzir a incerteza sobre as exposições reais em situação de trabalho, tornando-as visíveis [3].

16 A aposta passa a ser, então, a de caraterizar as situações de trabalho incluindo as atividades implicando exposição, com níveis de detalhe e escalas de tempo mais ou menos importantes em função das necessidades de transformação ou de conceção.

A abordagem inspira-se nos trabalhos precursores da ergonomia de conceção em torno da noção de " situação de acção característica " (SAC) como unidade de análise do trabalho para a concepção (Jeffroy, 1987 ; Garrigou, 1992 ; Daniellou, 1992 ; Garrigou et al., 1995 ; Daniellou, 2004 ; Duarte \& Lima, 2012 ; Barcellini et al., 2014), transposta em unidade de análise do trabalho exposto (a tóxicos) para conceber a prevenção graças às " situações de exposição características" (SEC) (Galey, Audignon, Witschger et al., 2019; Galey, Audignon, Witschgeret al., 2020). Esses resultados integram-se na concepção de uma " fábrica do futuro " implementando processos de fabricação aditiva de metais ou ainda numa tese pluridisciplinar entre o direito e a ergonomia para alterar o modo de conceção do material agrícola (Albert \& Garrigou, 2019). Essa ergotoxicologia ancorada nos questionamentos sócio-técnicos e regulamentares contemporâneos, a partir das contribuições dos predecessores, permite finalmente construir a prevenção pela mobilização das medições e da atividade, reduzindo-lhes a incerteza.

Um questionamento coletivo atual diz respeito às possíveis evoluções do termo ergotoxicologia quando a finalidade é a de produzir conhecimentos sobre as atividades sujeitas à exposição (a produtos tóxicos) para as transformar, mas não conhecimentos diretamente ligados à periculosidade dos produtos. A denominação "ergoexpologia " poderia ser apropriada, sem esquecer que os conhecimentos sobre potenciais efeitos tóxicos se mantém centrais para definir as modalidades de contaminação dos trabalhadores e potenciais sintomas tais como as observáveis e técnicas de medição que decorram no terreno. De modo similar à ergotoxicologia, a "ergoexpologia" interessarse-ia por caracterizar as situações de exposição a perigos que podem encontrar os trabalhadores no decurso do seu trabalho quer esses perigos sejam, nomeadamente, de natureza química, física ou biológica. Nesse quadro, a "ergoexpologia" teria por objeto compreender as atividades de trabalho face a essas exposições para as prevenir e conceber sistemas de trabalho seguros.

19 Como para a ergotoxicologia, parece-nos que esta proposta constitui, todavia, uma possibilidade de subespecialização da ergonomia assim como um risco (mas também uma oportunidade) para uma compreensão ampliada do trabalho. Por isso convém lembrar que a ergotoxicologia (ou a ergoexpologia) são antes de tudo abordagens estruturadas pelos paradigmas fundadores da ergonomia da atividade destinados aos ergónomos e ao conjunto dos atores da saúde no trabalho confrontados com os perigos dos sujeitos a exposições profissionais.

Uma coisa é certa, a " ergonomia vintage " [4] aplicada aos novos contextos do trabalho tem ainda diante dela dias auspiciosos. 


\section{BIBLIOGRAFIA}

Albert, M., \& Garrigou, A. (2019). Une approche critique de la conception et des usages du matériel de pulvérisation des pesticides en viticulture. Actes du 54ème Congrès de la Société d'Ergonomie de Langue Française (SELF). Comment contribuer à un autre monde? Tours, France.

Barcellini, F., Van Belleghem, L., \& Daniellou, F. (2014). Design projects as opportunities for the development of activities. In P. Falzon (Éd.), Constructive Ergonomics (pp. 187-204). CRC Press. https://doi.org/10.1201/b17456-16

Béguin, P. (2004). Mondes, monde commun et versions des mondes. Bulletin de psychologie, 57(469), $45-48$.

Béguin, P. (2010). Conduite de projet et fabrication collective du travail : Une approche développementale [Habilitation à diriger des recherches]. Laboratoire EVS UMR 5600, Université Victor Segalen Bordeaux 2, Bordeaux, France.

Cassou, B., Huez, D., Mousel, M.-L., Spitzer, C., \& Touranchet-Hébrard, A. (Dirs., 1985). Les risques du travail. Pour ne pas perdre sa vie à la gagner. Paris : La Découverte.

Daniellou, F. (1992). Le statut de la pratique et des connaissances dans l'intervention ergonomique de conception [Habilitation à diriger des recherches]. Laboratoire d'ergonomie des systèmes complexes, Université Victor Segalen-Bordeaux 2, Bordeaux, France.

Daniellou, F. (2004). 21. L'ergonomie dans la conduite de projets de conception de systèmes de travail. In P. Falzon, Ergonomie (1r éd., pp. 359-373). Paris : Presses Universitaires de France. https://doi.org/10.3917/puf.falzo.2004.01.0359

Daniellou, F. (2005). The French-speaking ergonomists' approach to work activity: Crossinfluences of field intervention and conceptual models. Theoretical Issues in Ergonomics Science, 6(5), 409-427. https://doi.org/10.1080/14639220500078252

Daniellou, F., Simard, M., \& Boissières, I. (2011). Human and organizational factors of safety : State of the art (Foundation for an Industrial Safety Culture). Toulouse : FonCSI.

DARES (2019). Comment ont évolué les expositions des salariés du secteur privé aux risques professionnels sur les vingt dernières années? Premiers résultats de l'enquête Sumer 2017 ( $\left.\mathrm{N}^{\circ} 41\right)$. Paris : Ministère du travail.

Delvolvé, N. (1984). Ergonomie et toxicologie. Le Travail Humain, 47(3), 227-235.

Deveau, M., Chen, C.-P., Johanson, G., Krewski, D., Maier, A., Niven, K. J., Ripple, S., Schulte, P. A., Silk, J., Urbanus, J. H., Zalk, D. M., \& Niemeier, R. W. (2015). The Global Landscape of Occupational Exposure Limits-Implementation of Harmonization Principles to Guide Limit Selection. Journal of Occupational and Environmental Hygiene, 12(sup1), S127-S144. https://doi.org/

10.1080/15459624.2015.1060327

Duarte, F., \& Lima, F. (2012). Anticiper l'activité par les configurations d'usage : Proposition méthodologique pour conduite de projet. Activités, 09(2). https://doi.org/10.4000/activites.314

Duclos, D. (1987). La construction sociale du risque : Le cas des ouvriers de la chimie face aux dangers industriels. Revue française de sociologie, 28(1), 17-42. https://doi.org/10.2307/3321444

Eurofound (2017). 6th European working conditions survey. Overview report [Publications Office of the European Union]. Bruxelles : Eurofound. 
Galey, L. (2019). Comprendre les situations d'exposition aux nanoparticules par l'intégration de l'activité de travail à la mesure : Vers une construction de la prévention [Thèse de doctorat]. Ecole doctorale Sociétés, Politique, Santé Publique, Université de Bordeaux, Bordeaux, France.

Galey, L., Audignon, S., Brochard, P., Debia, M., Lacourt, A., Lambert, P., Bihan, O., Martinon, L., Pasquereau, P., Witschger, O., \& Garrigou, A. (2020). Towards an operational exposure assessment strategy to airborne nanoparticles by integrating work activity analysis and exposure measurement. Archives des Maladies Professionnelles et de l'Environnement, 81(3). https://doi.org/ 10.1016/j.admp.2020.03.831

Galey, L., Audignon, S., Witschger, O., Bau, S., Judon, N., Lacourt, A., \& Garrigou, A. (2020). What does ergonomics have to do with nanotechnologies? A case study. Applied Ergonomics, 87(103116). https://doi.org/10.1016/j.apergo.2020.103116

Galey, L., Audignon, S., Witschger, O., Lacourt, A., \& Garrigou, A. (2019). Toward a Better Assessment of Occupational Exposure to Nanoparticles Taking into Account Work Activities. In S. Bagnara, R. Tartaglia, S. Albolino, T. Alexander, \& Y. Fujita (Éds.), Proceedings of the 20th Congress of the International Ergonomics Association (IEA 2018) (pp. 465-478). Springer International Publishing. https://doi.org/10.1007/978-3-319-96089-0_50

Galey, L., Audignon-Durand, S., Witschger, O., Lacourt, A., \& Garrigou, A. (2017). Intégration de la sécurité dans l'innovation : Vers une meilleure caractérisation des expositions professionnelles aux nanoparticules? Actes du 52e congrès de la SELF (pp. 169-173), Toulouse, France.

Galey, L., Judon, N., Jolly, C., Goutille, F., Morelot, S., Albert, M., Lhospital, O., Martin, P., NoelSuberville, C., Pasquereau, P., Aublet-Cuvelier, A., Mohammed-Brahim, B., \& Garrigou, A. (2019). Proposition méthodologique en ergotoxicologie pour révéler les expositions à des produits chimiques. Activités, 16(1). https://doi.org/DOI :10.4000/activites.4103

Garrigou, A. (1992). Les apports des confrontations d'orientation socio-cognitives au sein de processus de conception participatifs : Le rôle de l'ergonomie [Thèse de doctorat]. Laboratoire d'ergonomie, CNAM, Paris, France. http://www.theses.fr/1993CNAM0160

Garrigou, A. (2011). Le développement de l'ergotoxicologie : Une contribution de l'ergonomie à la santé au travail [Habilitation à Diriger des Recherches]. INSERM U1219, Université Victor Segalen Bordeaux 2, Bordeaux, France.

Garrigou, A., Baldi, I., Le Frious, P., Anselm, R., \& Vallier, M. (2011). Ergonomics contribution to chemical risks prevention: An ergotoxicological investigation of the effectiveness of coverall against plant pest risk in viticulture. Applied Ergonomics, 42(2), 321-330. https://doi.org/10.1016/ j.apergo.2010.08.001

Garrigou, A., Daniellou, F., Carballeda, G., \& Ruaud, S. (1995). Activity analysis in participatory design and analysis of participatory design activity. International Journal of Industrial Ergonomics, 15(5), 311-327. https://doi.org/10.1016/0169-8141(94)00079-I

Garrigou, A., Laurent, C., Berthet, A., Colosio, C., Jas, N., Daubas-Letourneux, V., Jackson Filho, J.M., Jouzel, J.-N., Samuel, O., Baldi, I., Lebailly, P., Galey, L., Goutille, F., \& Judon, N. (2020). Critical review of the role of PPE in the prevention of risks related to agricultural pesticide use. Safety Science, 123(104527). https://doi.org/10.1016/j.ssci.2019.104527

Garrigou, A., Peeters, S., Jackson, M., Sagory, P., \& Carballeda, G. (2004). 30. Apports de l'ergonomie à la prévention des risques professionnels. In P. Falzon, Ergonomie (1r éd., pp. 497-514). Paris : Presses Universitaires de France. http://www.cairn.info/ ergonomie--9782130514046-page-497.htm 
Garrigou, A., Théry, L., Chassaing, K., Effantin, E., Mercieca, P., Dimerman, S., Vanderghote, M., Negroni, P., Gauthier, P., Goutille, F., Galey, L., Rambaud, C., Laporte, E., Merlin, X., Vergneaux, L., \& Baratta, R. (2015). Une approche pluridisciplinaire du processus de construction sociale de la prévention du risque CMR. Actes du 50ème congrès de la SELF (pp. 476-489), Paris, France.

Goutille, F., Galey, L., Rambaud, C., Pasquereau, P., Jackson, M., \& Garrigou, A. (2016). Prescrição e utilização de equipamentos de proteção individual (EPI) em atividades com exposição a produtos químicos cancerígenos, mutagênicos e reprotóxicos (CMR) : Pesquisa-ação pluridisciplinar em uma fábrica francesa de decoração para móveis. Laboreal, 12(1). https://doi.org/10.15667/ laborealxii0116fg

Henry, E. (2000). Un scandale improbable. Amiante : D’une maladie professionnelle à une “ crise de santé publique" [Thèse de doctorat]. Université de Technologie de Compiègne.

INRS, \& ISSA. (2016). ED 6254Valeurs limites d'exposition pour la prévention des risques chimiques (pp. 82). Retrieved from http://www.inrs.fr/media.html?refINRS=ED\%206254

Jeffroy, F. (1987). Maîtrise de l'exploitation d'un système micro-informatique par des utilisateurs noninformaticiens : Analyse ergonomique et processus cognitif [Thèse de doctorat]. Paris 13, Paris, France.

Jouzel, J.-N. (2019). Pesticides. Comment ignorer ce que l'on sait. Paris : SciencesPo Les Presses.

Judon, N. (2017). Rendre possible un espace intermédiaire de dialogue pour co-construire de nouvelles solutions de prévention dans un contexte d'incertitude. Cas des travaux de revêtements routiers [Thèse de doctorat]. Ecole doctorale Sociétés, Politique, Santé Publique, Université de Bordeaux, Bordeaux, France.

Judon, N., Galey, L., Saint Dizier de Almeida, V., \& Garrigou, A. (2019). Contributions of participatory ergonomics to the involvement of workers in chemical risk prevention projects. Work, 64(3), 651-660. https://doi.org/10.3233/WOR-193001

Judon, N., Hella, F., Pasquereau, P., \& Garrigou, A. (2015). Vers une prévention intégrée du risque chimique lié à l'exposition cutanée au bitume des travailleurs de la route. Élaboration d'une méthodologie dans le cadre de l'ergotoxicologie. Perspectives interdisciplinaires sur le travail et la santé, 17(2). https://doi.org/10.4000/pistes.4586

Leplat, J., \& Cuny, X. (1977). Introduction à la psychologie du travail (1ère édition). Paris : PUF.

Mohammed-Brahim, B. (2015). L'ergotoxicologie ou comment aborder le risque chimique du point de vue de l'activité du travail. Actes de la troisième Conférence internationale sur Les applications de l'ergonomie dans les pays en voie de développement, 6. Alger, Algérie. Retrieved from http:// prevention-ergonomics.com/ar/Paper\%2014\%20Brahim\%20MOHAMMEDBRAHIM\%20\%20Ergo\%202015.pdf

Mohammed-Brahim, B., Daniellou, F., Brochard, P. (1997). “ Non pas que nous ne raisonnons pas, mais nous raisonnons autrement" : A propos d'un regard de l'ergonome sur la conduite raisonnée du traitement pesticide en viticulture. Actes du 32e congrès de la SELF (pp. 107-115), Lyon, France.

Mohammed-Brahim, B., Daniellou, F., Garrigou, A. (1998). Amiante: Chantier de retrait secteur 2. Guide pour le médecin du travail. pp. 125. Bordeaux : AHI 33.

Mohammed-Brahim, B., \& Garrigou, A. (2009). Une approche critique du modèle dominant de prévention du risque chimique. Activités, 06(1). https://doi.org/10.4000/activites.2086

Mohammed-Brahim, B., Garrigou, A., Treiber, A., Clement, N., Cuesta, C., Balent, I., Brun, G., \& Josse, P. (2018). Ergotoxicologie : État de l'art. Des pistes pour l'intervention à partir de retours d'expériences diversifiés. Actes du 53econgrès de la SELF (pp. 369-389), Bordeaux, France. 
Mollo, V., \& Falzon, P. (2004). Auto- and allo-confrontation as tools for reflective activities. Applied Ergonomics, 35(6), 531-540. https://doi.org/10.1016/j.apergo.2004.06.003

Morel, G., Amalberti, R., \& Chauvin, C. (2008). Articulating the Differences Between Safety and Resilience: The Decision-Making Process of Professional Sea-Fishing Skippers. Human Factors: The Journal of the Human Factors and Ergonomics Society, 50(1), 1-16. https://doi.org/ $10.1518 / 001872008 X 250683$

Nascimento, A., Cuvelier, L., Mollo, V., Dicioccio, A., \& Falzon, P. (2014). Constructing safety. In Constructive Ergonomics (pp. 95-110). CRC Press.

Reason, J. (1990). Human Error. New York: Cambridge University Press.

Rocha, R., Mollo, V., \& Daniellou, F. (2015). Work debate spaces: A tool for developing a participatory safety management. Applied Ergonomics, 46, 107-114. https://doi.org/10.1016/ j.apergo.2014.07.012

Sznelwar, L. I. (1992). Analyse ergonomique de l'exposition de travailleurs agricoles aux pesticides. Essai ergotoxicologique [Thèse de doctorat]. Laboratoire d'ergonomie, CNAM, Paris, France.

Teiger, C. (1993). L'approche ergonomique : Du travail humain à l'activité des hommes et des femmes au travail. Education Permanente, 3(116), 27.

Teiger, C., Laville, A., \& Gadbois, C. (2003). Robert Villatte. Entretien réalisé par Catherine Teiger et Antoine Laville (p. 9) [Commission Histoire de la Société d'Ergonomie de Langue Française].

Villatte, R. (1985). Toxicologie et ergonomie. InB. Cassou, D. Huez, M.-L. Mousel, C. Spitzer \& A. Touranchet-Hébrard (Dirs), Les risques du travail. Pour ne pas perdre sa vie à la gagner (pp. 301-303). Paris: La Découverte.

Vinck, D. (2009). From Intermediary Object towards Boundary-Object. Revue d'anthropologie des connaissances, 3(1), 51-72.

Weill-Fassina, A., Rabardel, P., \& Dubois, D. (Coord., 1993). Représentations pour l'action. Toulouse : Octarès.

Wisner, A. (1972). Diagnosis in Ergonomics or the Choice of Operating Models in Field Research. Ergonomics, 15(6), 601-620. https://doi.org/10.1080/00140137208924462

Wisner, A. (1985). Quand voyagent les usines. Essai d'anthropologie. Paris : Syros

Zartarian, V., Bahadori, T., \& McKone, T. (2004). Adoption of an official ISEA glossary. Journal of Exposure Science and Environmental Epidemiology. https://doi.org/10.1038/sj.jea.7500411

\section{NOTAS}

1. Poderíamos ainda interrogarmo-nos sobre o modo como as normas e barreiras de proteção constituem um modo de valorizar certos saberes sobre os riscos e não outros (Jouzel, 2019).

2. O termo «segurança» é utilizado com referência ao resultado global das ações de prevenção e uso das proteções em meio profissional. A utilização do termo é influenciada pela noção de segurança do trabalho ou ainda pelas pesquisas sobre segurança em meio industrial e hospitalar. Este termo «segurança» designa um trabalho «seguro», sem atentados à saúde, numa perspetiva durável e desenvolvimental.

3. A partir de medições e de vídeos da situação de trabalho, as suas análises e seleções em função de observações e hipótese subjacentes, os seus usos e colocação em circulação com os atores da empresa. 
4. Alain Garrigou denomina deste modo a ergotoxicologia em virtude das técnicas mobilizadas com aspetos tradicionais como a medição ou a análise da atividade.

\section{AUTORES}

\section{LOUIS GALEY}

Équipe TE2O, LAPPS (EA4386), Université Paris Nanterre, 200 avenue de la République, 92001 Nanterre Cedex, France

Équipe ergonomie, CRTD (EA4132) , Cnam, 41 rue Gay Lussac, 75005 Paris, France louis.galey@parisnanterre.fr

\section{ALAIN GARRIGOU}

EPICENE, U1219 INSERM Bordeaux Population Health Research Center, Université de Bordeaux, 146 rue Leo Saignat, 33076 Bordeaux Cedex, France

alain.garrigou@u-bordeaux.fr 